•生物编目・

\title{
神农架国家公园植物采集史及模式标本名录
}

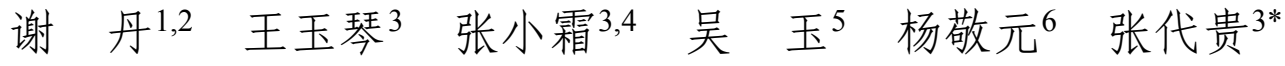 \\ 1 (中国科学院植物研究所系统与进化植物学国家重点实验室, 北京 100093) \\ 2 (中国科学院大学生命科学学院, 北京 100049) \\ 3 (吉首大学生物资源与环境科学学院, 湖南吉首 416000) \\ 4 (中国科学院昆明植物研究所, 云南昆明 650201) \\ 5 (湖南师范大学生命科学学院, 湖南长沙 410081) \\ 6 (神农架国家公园管理局, 湖北神农架 442421)
}

\begin{abstract}
摘要: 本文基于标本及文献数据对神农架国家公园高等植物标本采集史进行简要概述。该地区有着近140年的标 本采集历史, 最早对神农架地区进行植物标本采集的是爱尔兰植物学家Augustine Henry。中国科学院武汉植物园 标本馆(HIB)收藏了采自该地区的21,402份标本, 居首位; 其次为中国科学院植物研究所标本馆(PE) 16,483 份、华 中农业大学博物馆(CCAU) 1,851份。标本采集年份主要集中在1956-1959、1976-1981、1985-1987三个时间段, 其 中以1976年采集数量为最多。标本采集月份主要集中在4-11月, 8月采集量最大。不同植物的标本数量两极分化严 重, 标本量多的如桦叶荚蒾(Viburnum betulifolium), 高达 295 份, 然而有 612 种植物的标本量仅 1 份, 约 $2 / 3$ 的植物标 本量少于10份。因而, 在今后的采集工作中我们应该将重心置于标本数量不足的物种和类群, 而标本数量过剩的 类群则应减少或停止采集; 植物标本的采集过程应尽可能全覆盖, 突出对高海拔、峡谷、石壁、无人区等特殊生 境的调查。采集时间上应该注重春、冬二季。此外, 我们对该地区的维管植物模式标本进行了考证, 编制了《神 农架国家公园维管植物模式标本名录》。该地区共有维管植物模式标本39种2变种3变型, 隶属24科34属。对神农 架国家公园维管植物模式标本的整理为履行联合国教科文组织世界遗产中心的要求及进一步开展神农架遗产的 管理与保护提供了依据。
\end{abstract}

关键词: 分类学; 湖北; 模式标本; 新种; 标本馆

\section{A catalogue of plant type specimens and history of plant collecting in Shennongjia National Park}

Dan Xie ${ }^{1,2}$, Yuqin Wang ${ }^{3}$, Xiaoshuang Zhang ${ }^{3,4}$, Yu Wu ${ }^{5}$, Jingyuan Yang ${ }^{6}$, Daigui Zhang ${ }^{3 *}$

1 State Key Laboratory of Systematic and Evolutionary Botany, Institute of Botany, Chinese Academy of Sciences, Beijing 100093

2 College of Life Science, University of Chinese Academy of Sciences, Beijing 100049

3 College of Biology and Environmental Sciences, Jishou University, Jishou, Hunan 416000

4 Kunming Institute of Botany, Chinese Academy of Sciences, Kunming, Yunnan 650201

5 College of Life Sciences, Hunan Normal University, Changsha, Hunan 410081

6 Administration of Shennongjia National Park, Shennongjia, Hubei 442421

\begin{abstract}
Here we provide a brief overview of the history of higher plant collection in the Shennongjia National Park based on a literature review and specimen data. The local history of collecting dates back 140 years to when Irish botanist Augustine Henry first visited the area. Since then, 21,402 specimens collected in the Shennongjia region have been added to the herbarium of the Wuhan Botanical Garden, Chinese Academy of Sciences (HIB); 16,483 specimens are in the herbarium of the Institute of Botany, Chinese Academy of Sciences (PE) and 1,851 specimens are in the museum of Huazhong Agricultural University (CCAU). Most of the specimens were collected during three periods: 1956-1959, 1976-1981, and 1985-1987; with a peak
\end{abstract}

收稿日期: 2018-09-21; 接受日期: 2019-01-30

基金项目：湖北省技术创新重大专项(2018ACA132)、神农架金丝猴保育生物湖北省重点实验室开放性课题基金(2018SNJ0009)和国家标本资源共享平 台植物子平台项目(2005DKA21401)

* 通讯作者 Author for correspondence. E-mail: zdg634278@126.com 
of collecting in 1976. Most specimens were collected between April and November, with August being the month with maximum collection. The number of specimens of each varies greatly, from the 295 specimens of Viburnum betulifolium to a single specimen of 612 species; nearly $2 / 3$ of the species are represented by fewer than ten specimens. Thus, more effort should be focus on collecting insufficiently sampled taxa rather than on those already well represented. All areas should be covered as thoroughly as possible, especially difficult to reach habitats at high elevations, in valleys, on cliffs, and in less-populated areas. Additional fieldwork should be undertaken in less favorable growing seasons, such as in winter and early spring. Thirty nine species, two varieties, and three forms, representing 34 genera in 24 families have been described from Shennongjia. The checklist provides references for meeting the requirements for United Nations World Natural Heritage Site status and for its management and conservation.

Key words: taxonomy; Hubei; type specimens; new species; herbarium

神农架位于湖北西北部 $31^{\circ} 15^{\prime}-31^{\circ} 57^{\prime} \mathrm{N}$, $109^{\circ} 56^{\prime}-110^{\circ} 58^{\prime} \mathrm{E}$ 的区域, 由兴山县、房县、巴东县 的边缘部分组成。它地处我国西南高山(青藏高原余 脉一峨眉山)向东部低山丘陵过渡地带, 整个山体为 秦岭-大巴山脉的延伸, 平均海拔 $1,800 \mathrm{~m}$, 主峰神 农顶高达3,106.2 m, 下谷坪乡石柱河仅 $398 \mathrm{~m}$, 海 拔高差达 $2,708.2 \mathrm{~m}$ 。气候上具明显的东西过渡特征, 季风北迁亦途经此地, 加上特殊的地质环境, 该区 小气候特征显著。南北植物在此汇聚, 低海拔处分 布有部分亚热带物种, 高海拔处物种温带特征显著 (Bartholomew et al, 1983)。2011-2013年神农架开展 了第四次全国中药资源普查及本底调查, 共统计到 维管束植物 222 科 1,184 属3,550种(含种下单位), 其 中蒝类植物 38 科 85 属 289 种, 裸子植物 9 科 27 属 50 种, 被子植物 175科1,072属3,211种(谢丹等, 2017b)。此 外, 该地区植物区系起源古老, 连香树 (Cercidiphyllum japonicum)、水青树(Tetracentron sinense)、 领春木(Euptelea pleiosperma) 等第三纪子遗植物在 此有分布(杽大勇等, 2017)。同时，该地还有大量的 特有植物, 如征镒麻属 (Zhengyia) (Deng et al, 2013)、膜叶贯众(Cyrtomium membranifolium) (孔宪 需和王培善, 1997)、神农架紫苑(Aster shennongjiaensis) (Li \& Zhang, 2004)等。该区珍稀植物亦不 在少数, 如珙桐(Davidia involucrata)、红豆杉(Taxus wallichiana var. chinensis)、南方红豆杉(T. wallichiana var. mairei)等国家一级重点保护野生植物, 有 台湾水青冈(Fagus hayatae)、秦岭冷杉(Abies chensiensis)、鹅掌楸(Liriodendron chinense)等国家二级重 点保护野生植物。该地区拥有当今世界中纬度地区 保持完好的亚热带森林生态系统, 拥有大量的珍稀 动植物, 被列为联合国教科文组织“人与生物圈计
划” (MAB)成员之一(于倩等, 2008)。2016年7月 17 日在土耳其伊斯坦布尔举行的UNESCO世界遗产 委员会第40届会议上, 湖北神农架被正式列入 《世 界遗产名录》。

作为我国生物多样性热点地区之一，该区已经 开展了一系列的生物多样性研究(于倩等, 2008; 谢 宗强等, 2017; 周友兵等, 2017)。植物研究也引起了 较多的关注。标本是植物采集历史的记录者和传承 者, 能直接客观地反映植被状况、采集活动等信息。 本文基于标本数据以及文献资料对神农架地区植 物标本的采集情况进行了梳理, 以期详实了解该地 区植物标本的采集状况。

\section{资料收集与统计}

\section{1 数据来源及模式标本处理}

数字化标本信息主要来源于中国数字植物标 本馆(CVH, http://www.cvh.ac.cn/)、国家标本资源共 享平台(NSII, http://www.nsii.org.cn/2017/home.php) 以及JSTOR (https://plants.jstor.org/)和GBIF (https:// www.gbif.org/)。文献方面的数据主要来源于中国知 网(http://www.cnki.net/)、百度学术(http://xueshu.bai du.com/)、BHL (http://www.biodiversitylibrary.org/) 和Botanicus (http://www.botanicus.org/)等数据库。此 外，还查阅了Bretschneider (1898)编制的History of European Botanical Discoveries in China以及 《中国 植物采集简史》(王印政等, 2004)等描述我国植物采 集历史的书籍。数据收集截止于 2018 年 4 月 10 日, 共 获得1930-2015年间采自神农架地区的45,552条标 本数据(附录 1 )。

借助The International Plant Names Index (IPNI) (http://www.ipni.org/)搜索模式标本采自湖北的植物 
名称，对采自神农架的模式种一一进行考证; 查阅 Bartholomew (1983)在Journal of the Arnold Arboretum 中记录的中美联合鄂西考察采集的物种以及 Sargent (1917)在Plantae Wilsonianae 中记载的早期 Wilson和Henry采自鄂西的物种, 对采自该区域的 模式种进行梳理和统计。

\section{2 数据标准化及统计分析}

数据的标准化整理尤为重要。同号标本在不同 标本馆经过不同人的数字化整理, 常会出现不同的 数据条; 甚至同一个人记录同一个事件, 有时前后 亦会有所差别。如1980年的中美联合考察就有多种 录入格式(中美联合鄂西考察队、中美考察队、中美 神农架植物考察队、中美联合鄂西植考队、中美联 合鄂西植物考察队、Sino-Amer. Bot. Exped., 1980、 Sino-American Botanical Expedition等); 鄂神农架 植考队就有鄂神考队、鄂神农架队、鄂神农架植改 队等近 70 种名称。此外, 同一采集人、同一采集号 的标本经不同人鉴定也会出现不同的鉴定结果, 可 能是该采集号包括两种极其相近的植物而混淆所 致, 也有可能是其他人为因素导致。如Nowicke将 1980 Sino-Amer. Bot. Exped. No. 723号标本鉴定为 Phytolacca cf. polyandra Batalin (Bartholomew et al, 1983)。从 (Phytolacca acinosa) 的，也有鉴定为多雄荵商陆 $(P$. polyandra $)$ 的。然而, Xie等(2017)研究发现, 该种实 为商陆属一新种鄂西商陆(Phytolacca exiensis)。

个人采集则相对较好, 通常记录为中文全称或 英文缩写, 如吴鹏程(P. C. Wu)、张宪春(X. C. Zhang) 等; 但常因字体复杂、书写潦草造成记载错误。如 傅国勋常写成付国勋、付口勋、付国勳等; 石世贵 写成石世贡、石州贵等。我们对1930-2015年间采 自神农架地区的 45,552 条标本数据基于现有数据资 料库 $(\mathrm{CVH}, \mathrm{IPNI})$ 对采集人/队进行了标准化整理, 基于《中国生物物种名录(植物卷)》(中国生物物种 名录编委会, 2013-2018)、TROPICOS (http://www. tropicos.org/)及The Plant List (http://www.theplantlist. org/)等对拉丁学名进行校正。我们利用处理过的数 据对神农架标本在各地馆藏情况、不同年月植物标 本采集情况、不同类群标本采集情况及不同阶段主 要植物采集活动进行了分析。图表均在OriginPro 2017中完成。

\section{结果}

\section{1 不同年月标本采集情况}

规范采集日期后共获得44,089条记载有详细采 集日期的标本数据, 另有 218 条记录了年月，152条 仅记载年, 剩余1,093条未记载日期。对采集年份进 行分析发现，标本采集主要集中在1956-1959年、 1976-1981年、1985-1987年三个时间段(图1)，我们 通过查阅相关资料发现这三个阶段均有较为大型 的标本采集活动, 如神农架植物考察、1980中美联 合考察以及神农架作物种质资源调查等。尤以 1976 年采集规模最大, 鄂神农架植考队在这一时期采集 了2万余份标本, 其余年份多为零散采集。从采集月 份来看(图2), 标本采集主要集中在4-11月,8月采集 量最大; 而12月几乎没有采集记录, 1-3月也仅有骞 胗数份标本。这与植物物候有着密切的关系。标本 常要求具花或果等重要识别特征, 植物花果期主要 集中在夏、秋二季，因而该时期常为标本采集的高 峰段。相反, 冬、春二季气候较为寒冷, 多数草本 植物枯萎, 部分木本植物落叶, 因而能够采集的标 本数量偏少, 植物考察活动也偏少, 但该季节仍是 植物采集过程中不容忽视的一个时段。如孙航通泉 草(Mazus sunhangii) (Deng et al, 2016), 神农架淫羊 蕉(Epimedium shennongjiaensis) (Zhang \& Li, 2009) 等众多植物种类正是在该时间段处于生长繁殖期。

\section{2 不同类群标本采集情况}

对植物学名进行初步整理后得到43,596条鉴定 到种的数据, 另有 265 条鉴定到属、 1,431 条鉴定到 科、260条未鉴定。对数据去重并基于《中国生物

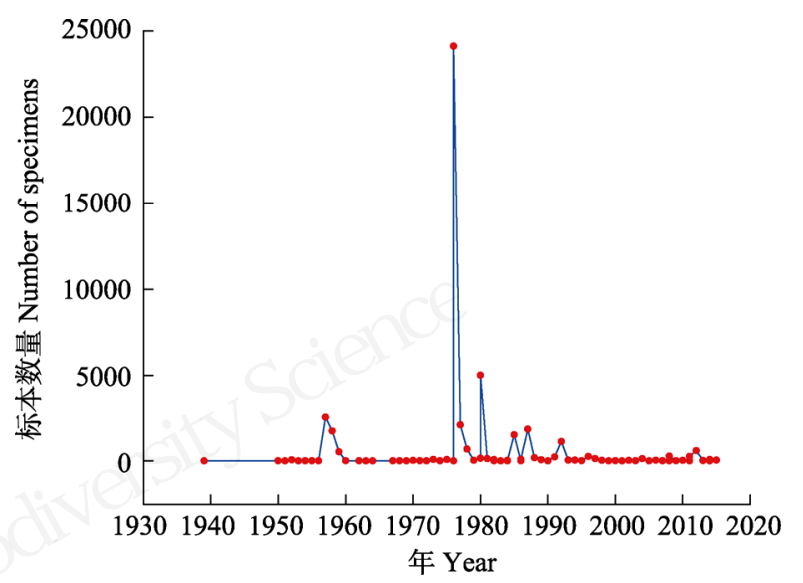

图1 神农架标本采集年份统计

Fig. 1 Number of specimen collected in different years in Shennongjia National Park 


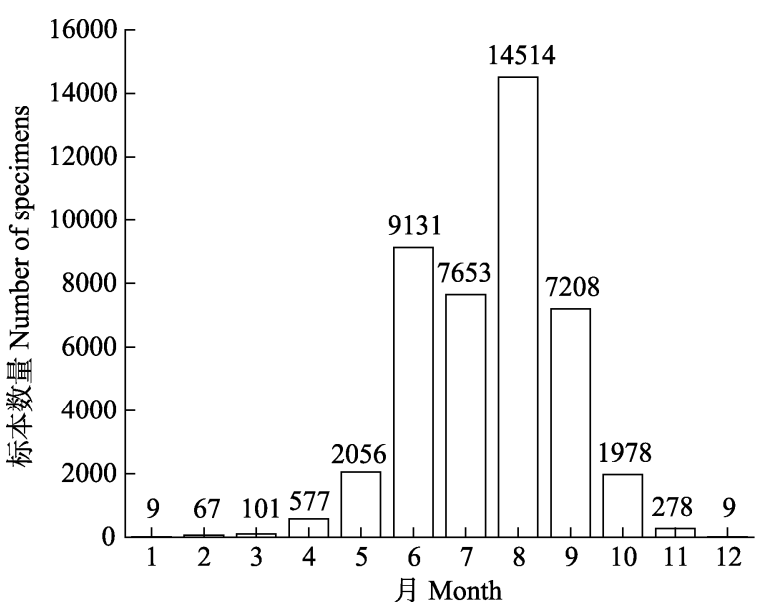

图2 神农架标本采集月份统计

Fig. 2 Number of specimen collected in different months in Shennongjia National Park

物种名录(植物卷)》进行学名校正, 共获得 3,040 条 接受名, 学名对应 218 科939属。结果显示该区域标 本采集量最多的科为蓄薇科, 标本数高达 3,633 份; 其次为菊科 2,219 份、毛茛科 1,291 份。标本采集量 前三的属依次为荚蒾属(Viburnum) 985份、忍冬属 (Lonicera) 642份、卫矛属(Euonymus) 557份。16种 植物的标本采集量超过 100 份, 其中采集量最多的 种为桦叶荚蒾 (Viburnum betulifolium) 295份, 其次 为卫矛(Euonymus alatus) 182份、蜡子树(Ligustrum leucanthum) 145份; 159种植物的标本采集量逾50份; 采集量在 10 份以上的物种数量占据全部物种的 $1 / 3$ $(1,085 / 3,040) ;$ 近 $2 / 3$ 的物种标本数量少于 10 份, 有 612 种标本采集量仅 1 份。标本采集数量多的类群或 者物种通常占据较广的分布区, 它们更容易为采集 者所关注。

\section{3 标本馆馆藏情况}

从目前收录的标本数据记录来看, 中国科学院 武汉植物园以 21,402 份居首位, 其次为中国科学院 植物研究所标本馆(PE，16,483)和华中农业大学博 物馆(CCAU, 1,851)。阿诺德植物标本馆(A)、纽约 植物园标本馆 $(N Y)$ 、爱丁堡植物园标本馆(E)及密苏 里植物园标本馆(MO)等也馆藏了部分采自该地的 标本。这些标本多为1980年中美联合鄂西考察时所 采集。具体馆藏情况见附录2。标本的采集对了解 地区植被及其分类情况提供了第一手资料, 为后续 研究打下了良好的基础。

\section{4 标本模式考证}

基于标本数据以及文献信息对模式标本采自
神农架地区的物种进行统计，我们编制了《神农架 国家公园维管植物模式标本名录》(附录3)。Sargent (1917)在Plantae Wilsonianae 中记录了大量Wilson和 Henry在鄂西考察时采集的标本, 其中不乏新物种, 书中记载了较多模式标本采自房县(Fang Hsien)的 物种, 如精柄茏荕(Smilax trachypoda)、房县柳(Salix rhoophila)、多枝柳(S. polyclona) 等; 模式标本采自 兴山(Hsing-shan)的物种, 如椅杨(Populus wilsonii)、 兴山柳(Salix mictotricha)、兴山五味子(Schisandra incarnate)等; 模式标本采自巴东县(Patung Hsien [Badong Xian])的巴柳(Salix etosia)、珊瑚朴(Celtis julianae)、巴东风毛菊(Saussurea henryi)等; 还有较 多没有详细地址, 统记为鄂西, 如E. H. Wilson n. 2061 的鄂西虎耳草(Saxifraga unguipetala)、A. Henry 7690 的湖北紫珠 (Callicarpa gracilipes) 以及 A. Henry 4725的鄂西天胡荌(Hydrocotyle wilsonii) 等。

神农架林区于1970年经国务院批准建制, 是中 国唯一以林区命名的县级行政区，由巴东县、房县、 兴山县三县的边缘地带组成。由于之前的标本采集 缺乏详细的记录, 因此较多之前采自巴东、房县、 兴山三县的模式标本是否属于神农架林区已无从 考证。本文仅对文献可考据且模式标本采于神农架 地区的物种进行统计, 共涉及39种2变种3变型, 隶 属24科34属(见附录3)。

从模式标本所属科来看, 以鳞毛偋科为最多, 发表新种 8 个; 其次为菊科(3种)、蹄盖颜科(3种); 水 龙骨科、小檗科、玄参科、毛茛科、蓄薇科各 2 种; 蜡 梅科发表 1 新变种 1 新变型, 其他各科均为 1 种;

从主模式所存放标本馆来看, PE以 22 份居首位, 其次为HIB (4份)、KUN (4份)、WUBI (2份)、湖南 农业大学理学院生物技术系标本室(2份), 其他各标 本馆各占1份。

从采集年份和采集人员来看, 1980年的中美联 合考察采集新种15种，其次是1976-1977年的神农 架植物考察队 8 种)以及神农架本底资源调查(3种)。

从原始文献所在期刊及志书来看, 主要为 Journal of the Arnold Arboretum (14种), 《植物研究》 (5种), 《植物分类学报》(4种), Phytotaxa (4种); Novon、《云南植物研究》、《湖南农业大学学报》、 《中国植物志》各描述2种; 其他如 Taxon、PLoS ONE等期刊亦有神农架地区新种的描述。

这些新物种的发现为之后的生物多样性研究 
提供了基础数据，同时模式标本的整理为神农架世 界自然遗产地履行联合国教科文组织世界遗产中 心的要求, 进一步开展神农架遗产的管理与保护提 供了依据。

\section{采集简史及讨论}

结合前人的研究结果, 通过分析大量的标本及 文献资料, 我们对神农架地区的植物采集历史进行 了简要的补充。早在1983年, Bartholomew在中美联 合科考报告中对神农架植物标本采集史进行了简 要概述。书中记载最早对神农架地区进行植物采集 的是爱尔兰的植物学家Augustine Henry。1882-1888 年, 他作为中国海事官员驻扎于湖北宜昌, 期间采 集了大量的植物标本。其中部分标本可能是由他雇 佣的中国工人所采集, 他本人的采集范围大致在以 宜昌为中心, 半径为 10-15英里的区域内 (Bretschneider, 1898)。1888年, 他在去保康县、房县、巫山 县的旅行途中, 抵达了一处海拔为 10,000 英尺(约 $3,000 \mathrm{~m})$ 的地方, 有绵延不断的高山和丰富的植被, 没有外国人, 甚至没有罗马天主教传教士曾到达过 (Henry in Thiselton-Dyer, 1889, p. 226)。从他写给英 国皇家植物园邱园馆长的信笺以及所提及的植物 来看, 可以大致推测该地区为神农架。第二个抵达 该区域的是英国植物学家E. H. Wilson。早在1900年, 他就开始了在湖北西部及四川东部的标本采集工 作。他有过4次在中国考察的经历, 从他第一次考察 的旅行地图我们可以看出他曾抵达过现神农架地 区。同时在Wilson (1913)的著作 $A$ Naturalist in Western China 中提及了大龙潭、小龙潭、大九湖等 现今属于神农架的地区。

我国学者从 20 世纪 20 年代开始神农架的植物 学考察。陈焕锯、钱崇澍和秦仁昌为我国最早对该 区域进行标本采集的植物学家。1922年7月, 他们离 开宜昌县前往兴山西部的万朝山、小龙潭等地, 历 经 2 个月采集了 1,000 多号植物标本。然而不幸的是, 存放在上海招商局仓库中最完善的一套标本于 1924年失火被焚, 其他复份零散存放于南京大学植 物标本馆 $(\mathrm{N})$ 、中国科学院植物研究所标本馆 $(\mathrm{PE})$ 、 阿诺德植物标本馆(A)、美国国家植物标本室(US)。 Y. Chen于1926年采集了将近3,000号标本, 其中部 分采自神农架林区, 由于采集记录的遗失, 具体地 点无从考证。这些标本存放于南京大学及南京林业
大学树木标本室(NF)。1930-1950期间有些许零散 的标本采集记录。1943年7至8月, 神农架开展了一 次林业考察, 王战作为其中一员参加了去万县和磨 刀溪的考察, 正是在这次考察中他发现了古老珍稀 子遗“活化石”植物一一水杉(Metasequoia glyptostroboides), 成为第一个采集到水杉标本的植物学家; 该区域后来被胡秀英总结为“水杉植物区系”, 吸引 了一大批国外学者, 如R. W. Chaney和J. L. Gressitt 等。1950-1976年期间有几次较小规模的标本采集 行动, 如傅国勋、李洪钧、钱敏之、黄仁煌、赵子 恩、陈封怀以及吕志松等, 详细采集情况见附录 4 。

1976-1978年, 神农架植物科考队对该地区的 植被和植物资源进行了初步调查, 采集了 10,000多 号标本, 主要存放于中国科学院武汉植物园标本馆 (HIB)和中国科学院植物研究所标本馆(PE), CVH记 录有 8,784 号 16,795 份，约 $90 \%$ 的标本采于 1976 年; “236-6”部队在该阶段亦采集了一定量的标本, 其 中1976年标本采集数量达973份，同年吴鹏程在该 地采集苔藓标本457份。1980年, 中美植物学家联合 对鄂西进行了为期 3 个月的植物考察 $(8$ 月 15 日至 11 月 15 日), 这是自 1949 年建国以来美国植物学家首 次到中国进行考察, 这次考察采集了大量的植物标 本并收集了部分种质资源(Bartholomew et al, 1983; Dosmann \& Tredici, 2005)。6周的野外工作共采集标 本 2,085 号, 其中维管植物 1,715 号, 全套标本存放 于中国科学院武汉植物园(HIB), CVH收录有 1,344 号2,034份; 其次为阿诺德植物标本馆(A) 1,695号 (CVH收录5号5份; GBIF收录46号51份); 中国科学 院植物研究所标本馆(PE) 1,605号(CVH收录 1,229 号 1,425 份); 中国科学院昆明植物研究所标本馆 (KUN) 1,431号(CVH收录596号600份); 江苏省中国 科学院植物研究所标本馆(NAS) 1,466号(CVH收录 78 号 82 份); 纽约植物园标本馆(The New York Botanical Garden, NY) 1,433号(JSTOR收录8号8份; GBIF收录17号23份); 爱丁堡植物园标本馆(Royal Botanic Garden, Edinburgh, E) 872号(JSTOR收录2 号2份; GBIF收录28号29份); 京都大学植物标本馆 (KYO) 454 号; 密苏里植物园标本馆 (Missouri Botanical Garden, MO) 173号(GBIF收录11号11份); 卡内基自然历史博物馆 (Carnegie Museum of Natural History, CM) 1,413号; 美国国家植物园(NA) 1,441号; 加利福尼亚大学植物标本馆(UC) 1,489号; 
武汉大学植物标本馆(WH) 1,199号; (SFDH) 1,309 号。这次考察过程发现 13 个新种, 1 个新变种及 1 个 新变型(Bartholomew et al, 1983)。1985年杨仕煊采 集植物标本 930 份, 刘少祥于1987年采集植物标本 905份，此后更有太阳坪考察队、陈龙清等采集了大 量标本。自2001年以来, 神农架开始作为武汉大学 野外科考实习基地, 迄今已采集标本 32,000余份(标 本数量由武汉大学汪小凡教授提供。标本数据尚未 上传至CVH或NSII网站, 因而未基于该数据进行统 计分析)。

在标本的采集过程中, 大量新物种及新分布被 发现，为了解该地的植被情况提供了良好的基础。 如沈泽昊和赵子恩(2005)基于其2001年于金丝燕垭 采集的8,594号标本发表了神农架无心菜(Arenaria shennongjiaensis); Li和Zhang (2004)基于 Wei-Ping Li 0776695发表了神农架紫苑, Chen等(2012)基于 Henry 1885-1888在鄂西地区采集的A. Henry 6,487 号发表了湖北蟹甲草(Parasenecio dissectus)等。 2011年神农架本底资源调查项目正式启动，这是该 地区一次较为全面的资源普查活动, 采集范围涉及 包括大面积无人区在内的各类生境, 采集时间尽可 能覆盖各个时段。历时 2 年采集了植物标本 6,660 号 (CVH收录的数据化标本约 726 号。已数字化的标本 数量与资料记载尚存在较大的出入)。这一阶段发现 了新属征镒麻属; 新种孙航通泉草、鄂西商陆等; 新记录科星叶草科(杨敬元等, 2013); 新记录属冷 蕨属(Cystopteris)、金线兰属(Anoectochilus)、双果 荠属(Megadenia)、顶冰花属(Gagea)、筒距兰属 (Tipularia) 等以及大量新记录种 (谢丹等, $2017 \mathrm{a}$, 2017b, 2018; 刘群等, 2017; 吴玉等, 2017)。

尽管在神农架地区已开展了数次大规模的植 物采集活动, 近年来仍有不少新物种被发现, 该地 区或仍有较多的物种有待研究。我们应在今后的工 作中加大对该地区的研究力度, 加强对这些物种的 保护工作, 为神农架世界自然遗产地保护的具体实 施提供科学建议(谢宗强等, 2017)。

标本数据清理仍是一项重要的任务。现有标本 虽然已经在很大程度上对数据进行了规范化整理, 但各类问题仍层出不穷，如采集人/队杂乱、采集日 期格式多样、鉴定力度不足等。物种采集上两极分 化严重, 多者可达数百份, 少者仅 1 份或无。文献资 料记载的标本数量与现有数字化标本量之间存在
较大的差距，这可能是海外标本信息无法获得、国 内标本数字化尚未完全所导致的。通过对标本数据 的整理和分析，我们发现了标本采集方面的不足， 这些信息为今后采集工作的开展提供了参考。采集 力度上我们应将重心置于标本数量不足的物种和 类群，尤其应该增加对模式标本的补充采集和调查， 并对物种模式产地进行定点保护; 而标本数量过剩 的类群则应减少或停止采集，确保区域内每种植物 均能在神农架标本室及该省从事分类学研究的权 威机构找到对应的标本。模式标本的数量与地区物 种多样性水平、研究力度、调查频次均有较大的关 系。物种多样性水平越高、研究力度越强、调查频 次越多, 记录的模式标本数量也越多。标本采集过 程中详细的原始记录显得尤为重要, 这是后续研究 的重要凭证。植物标本的采集过程应尽可能全覆盖, 突出对高海拔、峡谷、石壁、无人区等特殊生境的 调查、采集时间上应该注重春、冬二季，标本应尽 可能具备花、果等重要识别特征。

致谢：感谢中国科学院植物研究所覃海宁研究员、 张志耘研究员以及哈佛大学 David E Boufford 教授 对本文修改提出的宝贵意见!

\section{参考文献}

Bartholomew B, Boufford DE, Chang AL, Cheng Z, Dudley T, He SA, Jin YX, Li LY, Luteyn JL, Spongberg SA, Sun SC, Tang YC, Wan JX, Ying TS (1983) The 1980 Sino American Botanical Expedition to western Hubei Province, People's Republic of China, Journal of the Arnold Arboretum, 64, 1-99.

Bretschneider EV (1898) History of European Botanical Discoveries in China, Vol. I-II. Sampson Low, Marston and Company, London.

Chen YS (2012) A new species and a new combination in Parasenecio (Asteraceae). Annales Botanici Fennici, 48, 166-168.

Deng T, Kim C, Zhang DG, Zhang JW, Li ZM, Nie ZL, Sun H (2013) Zhengyia shennongensis: A new bulbiliferous genus and species of the nettle family (Urticaceae) from Central China exhibiting parallel evolution of the bulbil trait. Taxon, 62, 89-99.

Deng T, Zhang XS, Kim C, Zhang JW, Zhang DG, Volis S (2016) Mazus sunhangii (Mazaceae), a new species discovered in Central China appears to be highly endangered. PLoS ONE, 11, e0163581.

Dosmann MS, Tredici PD (2005) The Sino-American 
Botanical Expedition of 1980: A retrospective analysis of success. Hort Sciences, 40, 302-303.

Fan DY, Gao XM, Yang Y, Xiong GM, Shen GZ, Zhao CM, Xu WT, Zhou YB, Xie ZQ (2017a) Archaic nature of seed plants from Shennongjia World Heritage Site, China. Plant Science Journal, 35, 835-843. (in Chinese with English abstract) [樊 大勇, 高贤明, 杨永, 熊高明, 申国珍, 赵常明, 徐文婷, 周友兵, 谢宗强 (2017) 神农架世界自然遗产地种子植物 科属的古老性. 植物科学学报, 35, 835-843.]

Kong XX, Wang PS (1997) New materials for the Cyrtomium Presl of China. Chinese Journal of Applied and Environmental Biology, 3, 23-25. (in Chinese with English abstract) [孔宪需, 王培善 (1997) 中国贯众属的新资料. 应用与 环境生物学报, 3, 23-25.]

Li WP, Zhang ZG (2004) Aster shennongjiaensis (Asteraceae), a new species from Central China. Botanical Bulletin of Academia Sinica, 45, 95-99.

Liu Q, Xie D, Chen YX, Yang KH, Zhang DG (2017) Three newly recorded genera of Brassicaceae in Hubei. Acta Botanica Boreali-Occidentalia Sinica, 37, 1672-1676. (in Chinese with English abstract) [刘群, 谢丹, 陈庸新, 杨开 华, 张代贵 (2017) 湖北十字花科 3 新记录属. 西北植物 学报, 37, 1672-1676.]

Sargent CS (1917) Plantae Wilsonianae, Vol. 3. The University Press, Cambridge.

Shen ZH, Zhao ZE (2005) Arenaria shennongjiaensis, a new species of the Caryophyllaceae from Hubei, China. Acta Phytotaxonomica Sinica, 43, 73-75. (in Chinese with English abstract) [沈泽昊, 赵子恩 (2005) 湖北无心菜属 (石竹科)一新种——神农架无心菜. 植物分类学报, 43, 73-75.]

Species Catalogue of China Editorial Board (2013-2018) Species Catalogue of China: Volume Plants. Science Press, Beijing. (in Chinese) [中国生物物种名录编委会 (20132018) 中国生物物种名录(植物卷). 科学出版社, 北京.]

Wang YZ, Qin HN, Fu DZ (2004) A brief collection history of Chinese plants. In: Flora Reipublicae Popularis Sinicae, Tomus 1 (eds Wu ZY, Chen XQ), pp. 658-733. Science Press, Beijing. (in Chinese) [王印政, 覃海宁, 傅德志 (2004) 中国植物采集简史. 见: 中国植物志第一卷第六 章(吴征镒，陈心启编), 658-733页. 科学出版社, 北京.]

Wilson EH (1913) A Naturalist in Western China, Vol. 1. Methuen, London.

Wu Y, Pan HX, Duan XY, Zhang B, Xie ZX, Zhang DG (2017) Eight plant new records in Hubei Province. Journal of Yunnan Agricultural University (Natural Science), 32, 727-730. (in Chinese with English abstract) [吴玉, 潘茴香, 段晓云, 张博, 谢正新, 张代贵 (2017) 湖北植物新记录 8 种. 云南农业大学学报(自然科学), 32, 727-730.]

Xie D, Qian D, Zhang MH, Wang YQ, Wu Y, Huang LQ, Zhang DG (2017) Phytolacca exiensis, a new species of Phytolaccaceae from west of Hubei Province, China. Phytotaxa, 331, 224-232.
Xie D, Wu MH, Zhang B, Xun M, Zhang DG (2018) New record of ferns in Hubei Province. Guihaia, 38, 1480-1485. (in Chinese with English abstract) [谢丹, 吴名鹤, 张博, 寻敏, 张代贵 (2018) 湖北䓲类植物新记录. 广西植物, 38, 1480-1485.]

Xie D, Wu Y, Xiao JW, Xie ZX, Chen YX, He WQ, Zhang DG (2017a) New records of medicinal plants in Hubei. China Journal of Chinese Materia Medica, 42, 4436-4440. (in Chinese with English abstract) [谢丹，吴玉，肖佳伟，谢正 新, 陈庸新, 贺文琪, 张代贵 (2017a) 湖北药用植物新 记录. 中国中药杂志, 42, 4436-4440.]

Xie D, Zhang C, Zhang MH, Wu MH, Zhang DG (2017b) New records of monocotyledon plants in Hubei. Acta Botanica Boreali-Occidentalia Sinica, 37, 815-819. (in Chinese with English abstract) [谢丹, 张成, 张梦华, 吴名鹤, 张代贵 (2017b) 湖北单子叶植物新记录. 西北植物学报, 37 , 815-819.]

Xie ZQ, Shen GZ, Zhou YB, Fan DY, Xu WT, Gao XM, Du YJ, Xiong GM, Zhao CM, Zhu Y, Lai JS (2017) The outstanding universal value and conservation of the Shennongjia World Natural Heritage Site. Biodiversity Science, 25, 490-497. (in Chinese with English abstract) [谢 宗强, 申国珍, 周友兵, 㚞大勇, 徐文婷, 高贤明, 杜彦 君, 熊高明, 赵常明, 祝燕, 赖江山 (2017) 神农架世界 自然遗产地的全球突出普遍价值及其保护. 生物多样性, 25, 490-497.]

Yang JY, Yang KH, Liao MY, Zhou JJ, Chu ZF, Chen YX, Deng T, Zhang DG (2013) Circaeasteraceae-A new recorded angiosperm family from Hubei. Amino Acids \& Biotic Resources, 35(1), 25-27. (in Chinese with English abstract) [杨敬元，杨开华，廖明尧，周建军，储昭福，陈 庸新, 邓涛, 张代贵 (2013) 星叶草科——湖北被子植物 一新记录科. 氨基酸和生物资源, 35(1), 25-27.]

Yu Q, Xie ZQ, Xiong GM, Chen ZG, Yang JY (2008) Community characteristics and population structure of dominant species of Abies fargesii forests in Shennongjia National Nature Reserve. Acta Ecologica Sinica, 28, 1931-1941. (in Chinese with English abstract) [于倩, 谢宗 强, 熊高明, 陈志刚, 杨敬元 (2008) 神农架巴山冷杉 (Abies fargesii) 林群落特征及其优势种群结构. 生态学报, 28, 1931-1941.]

Zhang YJ, Li JQ (2009) A new species of Epimedium (Berberidaceae) from Hubei, China. Novon, 19, 567-569.

Zhou YB, Yu XL, Wu N, Shen GZ, Xiong GM, Xu WT, Fan DY, Zhao CM, Xie ZQ (2017) A catalogue of animal type specimens from the Shennongjia World Natural Heritage Site, China. Biodiversity Sciences, 25, 513-517. (in Chinese with English abstract) [周友兵, 余小林, 吴楠, 申国珍, 熊 高明, 徐文婷, 刹大勇, 赵常明, 谢宗强 (2017) 神农架 世界自然遗产地动物模式标本名录. 生物多样性, 25 , 513-517. ]

(责任编委：严岳鸿 责任编辑：时意专) 


\section{附录 Supplementary Material}

\section{附录1 神农架国家公园高等植物标本数据}

Appendix 1 Specimen data of higher plants from the Shennongjia National Park http://www.biodiversity-science.net/fileup/PDF/2018255-1.xlsx

\section{附录2 神农架国家公园高等植物标本馆藏单位}

Appendix 2 List of herbaria with specimens of higher plants from the Shennongjia National Park http://www.biodiversity-science.net/fileup/PDF/2018255-2.pdf

\section{附录3 神农架国家公园维管植物模式标本名录}

Appendix 3 Catalogue of vascular plant type specimens from the Shennongjia National Park http://www.biodiversity-science.net/fileup/PDF/2018255-3.pdf

\section{附录4 不同时段神农架国家公园主要标本采集人/队}

Appendix 4 Main collectors/teams of the specimen from Shennongjia National Park in different period of time http://www.biodiversity-science.net/fileup/PDF/2018255-4.pdf 
谢丹, 王玉琴, 张小霜, 吴玉, 张代贵. 神农架自然遗产地植物标本采集史. 生物多样性, 2019，27(2)：211-218. http://www. biodiversity-science. net/CN/10. 17520/biods. 2018255

附录 2 神农架国家公园高等植物标本馆藏单位

Appendix 2 Herbaria with specimens of higher plants from the Shennongjia National Park

\begin{tabular}{|c|c|}
\hline 标本馆 & 标本份数 \\
\hline Herbarium & Number of specimens \\
\hline \multicolumn{2}{|l|}{ 中国科学院武汉植物园标本馆 Herbarium, Wuhan Botanical Garden, Chinese Academy of Sciences } \\
\hline (HIB) & 21,402 \\
\hline 中国科学院植物研究所标本馆 Herbarium, Institute of Botany, Chinese Academy of Sciences (PE) & 16,483 \\
\hline 华中农业大学博物馆植物标本馆 Museum of Huazhong Agricultural University(CCAU) & 1,851 \\
\hline \multicolumn{2}{|l|}{ 中国科学院昆明植物研究所标本馆 } \\
\hline Herbarium, Kunming Institute of Botany, Chinese Academy of Sciences(KUN) & 951 \\
\hline \multicolumn{2}{|l|}{ 中国科学院华南植物园标本馆 } \\
\hline Herbarium, South China Botanical Garden, Chinese Academy of Sciences (IBSC) & 823 \\
\hline 吉首大学生物系植物标本室 Herbarium of Jishou university (JIU) & 729 \\
\hline 台湾林业研究所 Taiwan Forestry Research Institute (TAIF) & 547 \\
\hline \multicolumn{2}{|l|}{ 北京大学药学院中药标本馆 } \\
\hline Herbarium, Peking University School of Pharmaceutical Sciences(PEM) & 501 \\
\hline \multicolumn{2}{|l|}{ 广西植物研究所标本馆 Herbarium, Guangxi institute of botany, Chinese Academy of Sciences } \\
\hline (IBK) & 499 \\
\hline 江西省中国科学院庐山植物园标本馆 Herbarium, Lushan Botanical Garden (LBG) & 330 \\
\hline \multicolumn{2}{|l|}{ 西北农林科技大学生命科学学院植物研究所标本馆 } \\
\hline Herbarium, College of Life Sciences, Northwest Agriculture and Forestry University (WUK) & 328 \\
\hline \multicolumn{2}{|l|}{ 江苏省•中国科学院植物研究所标本馆 } \\
\hline Herbarium, Institute of Botany, Jiangsu Province and Chinese Academy of Sciences (NAS) & 246 \\
\hline \multicolumn{2}{|l|}{ 中国科学院成都生物研究所植物标本室 Herbarium, Institute of Biology, Chinese Academy of } \\
\hline Science (CDBI) & 145 \\
\hline 九江森林植物标本馆 Herbarium of Jiujiang Forestry Institute (JJF) & 105 \\
\hline 四川大学生物系植物标本室 Herbarium, College of Life Sciences, Sichuan University (SZ) & 100 \\
\hline 北京林业大学博物馆 Museum of Beijing Forestry University (BJFC) & 90 \\
\hline \multicolumn{2}{|l|}{ 中南林业科技大学林学院森林植物标本 Herbarium, Central South University of Forestry and } \\
\hline Technology (CSFI) & 83 \\
\hline 阿诺德植物标本馆 Herbarium of the Arnold Arboretum(A) & 56 \\
\hline \multicolumn{2}{|l|}{ 纽约植物园标本馆 The William and Lynda Steere Herbarium of the New York Botanical Garden } \\
\hline$(\mathrm{NY})$ & 40 \\
\hline 爱丁堡植物园标本馆 Royal Botanic Garden Edinburgh (E) & 34 \\
\hline 湖南科技大学植物标本室 Herbarium, Hunan University of Science and technology (HUST) & 31 \\
\hline \multicolumn{2}{|l|}{ 北京师范大学生命科学学院植物标本室 Herbarium, College of Life Sciences, Beijing Normal } \\
\hline University (BNU) & 31 \\
\hline 中国科学院沈阳应用生态研究所东北生物标本馆 (IFP) & 30 \\
\hline 陕西省西安植物园植物标本室 Herbarium, Xian Botanical Garden in Shanxi Province (XBGH) & 19 \\
\hline 密苏里植物园标本馆 Missouri Botanical Garden (MO) & 17 \\
\hline 上海辰山植物园标本室 Herbarium, Shanghai Chen Shan Botanical Garden (CSH) & 15 \\
\hline 加利福尼亚大学标本馆 University of California (UC) & 9 \\
\hline \multicolumn{2}{|l|}{ 中国科学院西北高原生物研究所植物标本馆 } \\
\hline Herbarium, Northwest Institute of Plateau Biology, Chinese Academy of Sciences (HNWP) & 8 \\
\hline \multicolumn{2}{|l|}{ 深圳市中国科学院仙湖植物园植物标本馆 } \\
\hline Herbarium, Fairylake Botanical Garden, Shenzhen \& Chinese Academy of Sciences (SZG) & 7 \\
\hline
\end{tabular}


谢丹, 王玉琴, 张小霜, 吴玉，张代贵．神农架自然遗产地植物标本采集史. 生物多样性，2019，27(2)：211-218. http://www. biodiversity-science. net/CN/10. 17520/biods. 2018255

\begin{tabular}{ll}
\hline 标本馆 & 标本份数 \\
Herbarium & Number of specimens \\
\hline 内蒙古大学生命科学学院植物标本馆 Herbarium, College of Life Science, Inner Mongolia & 5 \\
University (HIMC) & 4 \\
南京大学生物系植物标本室 Herbarium, School of Life Sciences, Nanjing University (N) & 4 \\
北京自然博物馆植物标本室 Herbarium, Beijing Museum of Natural History (BJM) & \\
中国科学院西双版纳热带植物园植物标本馆 & 3 \\
Herbarium, Xishuangbanna Tropical Botanical Garden, Chinese Academy of Sciences (HITBC) & 3 \\
邱园植物园 Royal Botanic Gardens (K) & 2 \\
英国自然历史博物馆 Natural History Museum (BM) & 2 \\
北京大学生物系植物标本室 Herbarium, Department of Biology, Peking University (PEY) \\
河南师范大学生命科学学院生物标本馆 Herbarium, College of Life Sciences, Henan Normal \\
University (HENU) \\
巴黎自然历史博物馆 Muséum National d'Histoire Naturelle (P) \\
格拉利茨森根堡自然博物馆 Herbarium Senckenbergianum Görlitz (GLM) \\
布拉格大学植物标本馆 Charles University in Prague Herbarium (PRC) \\
\hline
\end{tabular}


附录3 神农架国家公园维管植物模式标本名录

Appendix 3 Catalogue of vascular plant type specimens from the Shennongjia National Park

\begin{tabular}{|c|c|c|c|c|c|c|}
\hline $\begin{array}{l}\text { 序号 } \\
\text { No. }\end{array}$ & $\begin{array}{l}\text { 物种 } \\
\text { Species }\end{array}$ & $\begin{array}{l}\text { 科 } \\
\text { Family }\end{array}$ & $\begin{array}{l}\text { 原始文献 } \\
\text { Origin Literature }\end{array}$ & $\begin{array}{l}\text { 采集号 } \\
\text { Collect Number }\end{array}$ & 主模式 & 发表时间 \\
\hline 1 & 神农架铁线莲 Clematis shenlungchiaensis M. Y. Fang & 毛茛科 & $\begin{array}{l}\text { Flora Reipublicae Popularis Sinicae } \\
\text { 28: 355, 1980. (Wang et al, 1980) }\end{array}$ & Shennong. Exped. 10720 & PE & 1980 \\
\hline 2 & $\begin{array}{l}\text { 神农架唇柱芭苔 Chirita shennungjiaensis W.T. Wang } \\
=\text { = Chirita tenuituba (W.T. Wang) W.T. Wang] }\end{array}$ & 苦菅苔科 & $\begin{array}{l}\text { Bulletin of Botanical Research, Harbin } \\
\text { 1(4): 66-67, 1981. (Wang, 1981) }\end{array}$ & Shennong. Exped. 34256 & $\mathrm{~W}$ & 1981 \\
\hline 3 & 鄂西沙参 Adenophora hubeiensis D. Y. Hong & 桔梗科 & $\begin{array}{l}\text { Flora Reipublicae Popularis Sinicae } \\
\text { 73(2): 123, 186-187, 1983. (Hong et al, 1983) }\end{array}$ & Shennong. Exped. 31976 & $\mathrm{PE}$ & 1983 \\
\hline 4 & $\begin{array}{l}\text { Pyrrosia pseudocalvata Ching, Boufford, \& Shing } \\
\text { Pyrrosia calvata (Baker) Ching] }\end{array}$ & 水龙骨科 & $\begin{array}{l}\text { Journal of the Arnold Arboretum } \\
\text { 64(1): 38, 1983. (Bartholomew et al, 1983) }\end{array}$ & 1980 Sino-American Bot. Exp. 1100 & $\mathrm{PE}$ & 1983 \\
\hline 5 & $\begin{array}{l}\text { 鄂西绵果悬钩子 } \\
\text { Rubus lasiostylis var. hubeiensis Yu, Spongberg \& Lu }\end{array}$ & 蓄薇科 & $\begin{array}{l}\text { Journal of the Arnold Arboretum. } \\
\text { 64(1): 21, 1983. (Bartholomew et al, 1983) }\end{array}$ & 1980 Sino-American Bot. Exp. 114 & PE & 1983 \\
\hline 6 & $\begin{array}{l}\text { Pyrrosia caudifrons Ching, Boufford \& Shing } \\
=\text { Pyrrosia lingua } \text { (Thunberg) Farwell }\end{array}$ & 水龙骨科 & $\begin{array}{l}\text { Journal of the Arnold Arboretum } \\
\text { 64(1): 37, 1983. (Bartholomew et al, 1983) }\end{array}$ & 1980 Sino-American Bot. Exp. 1159 & PE & 1983 \\
\hline 8 & 长芒耳硕 Polystichum longiaristatum Ching, Boufford \& Shing & 鳞毛硕科 & $\begin{array}{l}\text { Journal of the Arnold Arboretum } \\
\text { 64(1): 33-34, 1983. (Bartholomew et al, 1983) }\end{array}$ & 1980 Sino-American Bot. Exp. 1248 & $\mathrm{PE}$ & 1983 \\
\hline 9 & $\begin{array}{l}\text { Dryopteris submarginalis Ching, Boufford \& Shing } \\
=\text { Dryopteris whangshangensis Ching] }\end{array}$ & 鳞毛鄀科 & $\begin{array}{l}\text { Journal of the Arnold Arboretum } \\
\text { 64(1): 30, 1983. (Bartholomew et al, 1983) }\end{array}$ & 1980 Sino-American Bot. Exp. 1356 & PE & 1983 \\
\hline 10 & $\begin{array}{l}\text { Lunathyrium vermiforme Ching, Boufford \& Shing } \\
=\text { Deparia vermiformis (Ching, Boufford \& K. H. Shing) Z.R. Wang }\end{array}$ & 蹄盖菜科 & $\begin{array}{l}\text { Journal of the Arnold Arboretum } \\
\text { 64(1): 23, 1983. (Bartholomew et al, 1983) }\end{array}$ & 1980 Sino-American Bot. Exp. 2025 & PE & 1983 \\
\hline 11 & $\begin{array}{l}\text { 华中蛾眉蕨 Lunathyrium shennongense Ching, Boufford \& Shing } \\
=\text { Deparia shennongensis (Ching, Boufford \& K. H. Shing) X. C. } \\
\text { Zhang] }\end{array}$ & 蹄盖蕨科 & $\begin{array}{l}\text { Journal of the Arnold Arboretum } \\
\text { 64(1): 21, 1983. (Bartholomew et al, 1983) }\end{array}$ & 1980 Sino-American Bot. Exp. 353 & PE & 1983 \\
\hline
\end{tabular}




\begin{tabular}{|c|c|c|c|c|c|c|}
\hline $\begin{array}{l}\text { 序号 } \\
\text { No. }\end{array}$ & $\begin{array}{l}\text { 物种 } \\
\text { Species }\end{array}$ & $\begin{array}{l}\text { 科 } \\
\text { Family }\end{array}$ & $\begin{array}{l}\text { 原始文献 } \\
\text { Origin Literature }\end{array}$ & $\begin{array}{l}\text { 采集号 } \\
\text { Collect Number }\end{array}$ & $\begin{array}{l}\text { 主模式 } \\
\text { Holotype }\end{array}$ & $\begin{array}{l}\text { 发表时间 } \\
\text { Publication time }\end{array}$ \\
\hline 12 & $\begin{array}{l}\text { Dryopteris apicifixa Ching, Boufford \& K. H. Shing } \\
=\text { Dryopteris fructuosa }(\text { Christ) C. Christensen }\end{array}$ & 鳞毛蕨科 & $\begin{array}{l}\text { Journal of the Arnold Arboretum } \\
\text { 64(1): 27-28. 1983. (Bartholomew et al, 1983) }\end{array}$ & 1980 Sino-American Bot. Exp. 543 & $\mathrm{PE}$ & 1983 \\
\hline 13 & 神农架冬青（Ilex shennongjiaensis T. R. Dudley \& S. C. Sun） & 冬青科 & $\begin{array}{l}\text { Journal of the Arnold Arboretum } \\
\text { 64(1): 63-65, 1983. (Bartholomew et al, 1983) }\end{array}$ & 1980 Sino-American Bot. Exp. 554 & $\mathrm{PE}$ & 1983 \\
\hline 14 & $\begin{array}{l}\text { Dryopteris infrapuberula Ching, Boufford, \& Shing } \\
\text { =Dryopteris namegatae (Sa. Kurata) Sa. Kurata }\end{array}$ & 鳞毛蕨科 & $\begin{array}{l}\text { Journal of the Arnold Arboretum } \\
\text { 64(1): 28-30, 1983. (Bartholomew et al, 1983) }\end{array}$ & 1980 Sino-American Bot. Exp. 619 & $\mathrm{PE}$ & 1983 \\
\hline 15 & $\begin{array}{l}\text { Athyrium amplissimum Ching, Boufford \& Shing } \\
=\text { Athyrium omeiense Ching }\end{array}$ & 蹄盖蕨科 & $\begin{array}{l}\text { Journal of the Arnold Arboretum } \\
64(1): 20,1983 \text {. (Bartholomew et al, 1983) }\end{array}$ & 1980 Sino-American Bot. Exp. 914 & $\mathrm{PE}$ & 1983 \\
\hline 16 & $\begin{array}{l}\text { Matteuccia orientalis f. monstra Ching \& Shing } \\
=\text { Pentarhizidium orientale } \text { (Hooker) Hayata }\end{array}$ & 球子蕨科 & $\begin{array}{l}\text { Journal of the Arnold Arboretum } \\
\text { 64(1): 25-26, 1983. (Bartholomew et al, 1983) }\end{array}$ & 1980 Sino-American Bot. Exp. 720 & $\mathrm{PE}$ & 1984 \\
\hline 17 & 神农架蒿 Artemisia shennongjiaensis Y. Ling \& Y. R. Ling & 菊科 & $\begin{array}{l}\text { Bulletin of Botanical Research, Harbin } \\
4(2): 24-25,1984 \text {. (Lin, 1984) }\end{array}$ & Shennong. Exped. 11832 & $\mathrm{PE}$ & 1984 \\
\hline 18 & 洪平杏 Armeniaca hongpingensis C. L. Li & 蓄薇科 & $\begin{array}{l}\text { Acta Phytotaxonomica Sinica 23: 209, } 1985 . \\
\text { (Yu et al, 1985) }\end{array}$ & Shennong. Exped. 34031 & WUBI & 1985 \\
\hline 19 & 神农架花楸 Sorbus yuana Spongberg & 蓄薇科 & $\begin{array}{l}\text { Journal of the Arnold Arboretum } \\
\text { 67(2): 257-261, 1986. (Spongberg et al, 1986) }\end{array}$ & 1980 Sino-American Bot. Exp. 1728 & A & 1986 \\
\hline 20 & 叉毛阴山荠 Yinshania furcatopilosa (K. C. Kuan) Y. H. Zhang & 十字花科 & $\begin{array}{l}\text { Acta Phytotaxonomica Sinica 25(3): 214. } 1987 . \\
\text { (Zhang, 1986) }\end{array}$ & Shennong. Exped. 21332 & $\mathrm{PE}$ & 1987 \\
\hline 21 & 鄂西黄堇 Corydalis shennongensis H. Chuang & 罂粟科 & $\begin{array}{l}\text { Acta Botanica Yunnanica } \\
\text { 12(3): 285-286, 1990. (Wu \& Zhuang, 19990) }\end{array}$ & 1980 Sino-American Bot. Exp. 399 & KUN & 1990 \\
\hline 22 & $\begin{array}{l}\text { 鄂西阴山荠 Yinshania exiensis Y.H.Zhang } \\
=\text { Yinshania zayuensis Y. H. Zhang] }\end{array}$ & 十字花科 & $\begin{array}{l}\text { Acta Botanica Yunnanica } \\
\text { 15(4): 364-365, 1996. (Zhang, 1996) }\end{array}$ & Shennong. Exped. 20597 & WUBI & 1996 \\
\hline 23 & 卵叶牡丹 Paeonia qiui Y. L. Pei \& D. Y. Hong & 药药科 & $\begin{array}{l}\text { Acta Phytotaxonomica Sinica } \\
\text { 33(1): 91-93.1995. (Pei \& Hong, 1995) }\end{array}$ & J.Z. Qiu PB88034 & $\mathrm{PE}$ & 1995 \\
\hline 24 & 木鱼坪淫羊雚 Epimedium franchetii Stearn & 小檗科 & Kew Bulletin 51(2): 396-398, 1996. & M. Ogisu 87001 & HT & 1996 \\
\hline
\end{tabular}




\begin{tabular}{|c|c|c|c|c|c|c|}
\hline 序号 & 物种 & 科 & 原始文献 & 采集号 & 主模式 & 发表时间 \\
\hline \multirow[t]{2}{*}{ No. } & Species & Family & Origin Literature & Collect Number & Holotype & Publication time \\
\hline & & & (William, 1996) & & & \\
\hline 25 & 膜叶贯众 Cyrtomium membranifolium Ching \& K. H. Shing & 鳞毛鄀科 & $\begin{array}{l}\text { Chinese Journal of Applied \& Environmental Biology } \\
\text { 3(01):23-25, 1997. (Kong and Wang, 2007) }\end{array}$ & Shennong. Exped. 33209 & $\mathrm{PE}$ & 1997 \\
\hline 26 & 神农岩茨 Woodsia shennongensis D. S. Jiang et D. M. Chen & 岩烣科 & $\begin{array}{l}\text { Journal of Hunan Agricultural University } \\
\text { 26(2):88,2000. (Jiang et al, 2000) }\end{array}$ & 蒋道松 (1992-08) 0083 & & 2000 \\
\hline 27 & 新正宇耳蕨 Polystichum neoliuii D. S. Jiang & 鳞毛蕨科 & $\begin{array}{l}\text { Journal of Hunan Agricultural University } \\
\text { 26(2):89, 2000. (Jiang et al, 2000) }\end{array}$ & 蒋道松 (1992-10) 0225 & & 2000 \\
\hline 28 & 神农架紫苑 Aster shennongjiaensis W.P.Li \& Z.G.Zhang & 菊科 & $\begin{array}{l}\text { Bulletin of Botanical Research, Harbin } 45(1): 96 \\
\text { (95-99), 2004. (Li \&Zhang, 2004) }\end{array}$ & Wei-Ping Li 0776695 & HNNU & 2004 \\
\hline 29 & $\begin{array}{l}\text { 神农架崖白菜 } \\
\text { Triaenophora shennongjiaensis Xiao D.Li, Y.Y.Zan \& J.Q.Li }\end{array}$ & 玄参科 & Novon 15(4): 559-561, 2005. (Li et al, 2005) & Yanyan Zan 238 & HIB & 2005 \\
\hline 30 & $\begin{array}{l}\text { 神农架无心菜 } \\
\text { Arenaria shennongjiaensis Z.E.Chao \& Z.H.Shen }\end{array}$ & 石竹科 & $\begin{array}{l}\text { Acta Phytotaxonomica Sinica 43(1): 73-75, } 2005 . \\
\text { (Shen and Zhao, 2005) }\end{array}$ & Z.H.Shen, Z.E.Zhao 8594 & HIB & 2005 \\
\hline 31 & $\begin{array}{l}\text { 卷瓣蜡梅 Chimonanthus praecox var. reflexus B. Zhao } \\
=\text { Chimonanthus praecox (Linnaeus) Link]） }\end{array}$ & 蜡梅科 & $\begin{array}{l}\text { Bulletin of Botanical Research, Harbin } \\
\text { 27(2): 131-132, 2007. (Zhao et al, 2007) }\end{array}$ & Z.B \& M X.Gong 121802 & PE & 2007 \\
\hline 32 & $\begin{array}{l}\text { 跳枝蜡梅 Chimonanthus praecox f. versicolor B. Zhao } \\
=\text { Chimonanthus praecox (Linnaeus) Link] }\end{array}$ & 蜡梅科 & $\begin{array}{l}\text { Bulletin of Botanical Research, Harbin } \\
\text { 27(2): 132, 2007. (Zhao et al, 2007) }\end{array}$ & Z.B \& M X.Gong 121813 & PE & 2007 \\
\hline 33 & 神农架乌头 Aconitum shennongjiaense Q.Gao \& Q.E.Yang & 毛莨科 & $\begin{array}{l}\text { Botanical Studies (Taipei) 50(2): 251-259, } 2009 . \\
\text { (Gao and Yang, 2009) }\end{array}$ & Qi Gao \& Y. S Chen 62 & PE & 2009 \\
\hline 34 & 卵叶卫矛 Celastrus obovatifolius X. Y. Mu \&Z. X. Zhang & 卫矛科 & $\begin{array}{l}\text { Nordic journal of botany. 30(1):53-57, } 2009 . \\
\text { (Mu et al, 2012) }\end{array}$ & Xian-Yun Mu 20081002 & BJFC & 2009 \\
\hline 35 & $\begin{array}{l}\text { 神农架淫羊雚 } \\
\text { Epimedium shennongjiaensis Yan J. Zhang \& J.Q.Li }\end{array}$ & 小檗科 & Novon 19(4): 567-569, 2009. (Zhang et al, 2009) & Y. J. Zhang 148 & HIB & 2009 \\
\hline 36 & 短茎蒲公英 Taraxacum abbreviatulum Kirschner \& Štěpánek & 菊科 & Flora of China 20-21: 306, 2011. (Ge et al, 2011) & JŠ 6344 & PRA & 2011 \\
\hline
\end{tabular}




\begin{tabular}{|c|c|c|c|c|c|c|}
\hline $\begin{array}{l}\text { 序号 } \\
\text { No. }\end{array}$ & $\begin{array}{l}\text { 物种 } \\
\text { Species }\end{array}$ & $\begin{array}{l}\text { 科 } \\
\text { Family }\end{array}$ & $\begin{array}{l}\text { 原始文献 } \\
\text { Origin Literature }\end{array}$ & $\begin{array}{l}\text { 采集号 } \\
\text { Collect Number }\end{array}$ & $\begin{array}{l}\text { 主模式 } \\
\text { Holotype }\end{array}$ & $\begin{array}{l}\text { 发表时间 } \\
\text { Publication time }\end{array}$ \\
\hline 37 & $\begin{array}{l}\text { 重瓣川鄂獐耳细辛 } \\
\text { Hepatica henryi f. pleniflora X. D. Li et J. Q. Li }\end{array}$ & 毛莨科 & $\begin{array}{l}\text { Acta Botanica Boreali-Occidentalia Sinica, } \\
\text { 31(11):2333-2334,2011. (Li et al, 2011) }\end{array}$ & Li 20080180 & HIB & 2011 \\
\hline 38 & 湖北耳䕋 Polystichum hubeiense Liang Zhang \& Li Bing Zhang & 鳞毛蕨科 & $\begin{array}{l}\text { Annales Botanici Fennici. } \\
\text { 50(1-2): 107, 2013. (Zhang et al, 2013) }\end{array}$ & Liang Zhang \& Zhang-Ming Zhu 1044 & CDBI & 2013 \\
\hline 39 & 征镒麻 Zhengyia shennongensis T.Deng, D.G.Zhang \& H.Sun & 菖麻科 & Taxon 62(1): 94, 2013. (Deng et al, 2013) & T. Deng, D.G. Zhang \& H. Sun 2295 & KUN & 2013 \\
\hline 40 & 神农凤仙花 Impatiens shennongensis Q.Wang \& H.P.Deng & 凤仙花科 & $\begin{array}{l}\text { Phytotaxa } 244 \text { (1): 96-100, } 2016 . \\
\text { (Wang et al, 2016) }\end{array}$ & Q.Wang 20130808 & SWU & 2015 \\
\hline 41 & 孙航通泉草 Mazus sunhangii D.G.Zhang \& T.Deng & 通泉草科 & $\begin{array}{l}\text { PLoS ONE 11(10): e0163581 (4), } 2016 . \\
\text { (Deng et al, 2016) }\end{array}$ & Dai-Gui Zhang et al. 4142 & KUN & 2016 \\
\hline 42 & 大苍冰岛苶 Koenigia hedbergii B. Li \& W. Du & 蓼科 & Phytotaxa 272 (2): 115-124, 2016. (Li et al, 2016) & W. Du 2129 & WH & 2016 \\
\hline 43 & 鄂西商陆 Phytolacca exiensis D.G.Zhang, L.Q.Huang \& D.Xie & 商陆科 & Phytotaxa 331(2): 227, 2017. (Xie et al, 2017b) & zdg10065 & JIU & 2017 \\
\hline 44 & 大齿玄参 Scrophularia jinii P. Li & 玄参科 & $\begin{array}{l}\text { Phytotaxa } 350 \text { (1): 001-014, } 2018 . \\
\text { (Wang et al, 2018) }\end{array}$ & LP150472 & $\mathrm{HZU}$ & 2018 \\
\hline
\end{tabular}


谢丹, 王玉琴，张小霜，吴玉，张代贵. 神农架自然遗产地植物标本采集史. 生物多样性，2019，27(2)：211-218. http://www. biodiversity-science. net/CN/10.17520/biods. 2018255

附录4 不同时段神农架国家公园主要标本采集人/队

Appendix 4 Main collectors/teams of the specimen from Shennongjia National Park in different period of time

\begin{tabular}{|c|c|c|c|c|c|}
\hline 年 & 采集人 & 份数 & 年 & 采集人 & 份数 \\
\hline Year & Collectors & Numbers & Year & Collectors & Numbers \\
\hline \multirow[t]{5}{*}{1957} & 傅国勋等 & 1267 & 1981 & 杜年生等 & 81 \\
\hline & 李洪均等 & 560 & 1982 & 蒋祖德\&陶光复 & 57 \\
\hline & 胡啟明 & 457 & 1985 & 杨仕煊 & 930 \\
\hline & 刘瑛 & 81 & & 姚习山 & 172 \\
\hline & 张志林等 & 57 & & 王清泉等 & 78 \\
\hline \multirow[t]{5}{*}{1958} & 黄仁煌等 & 581 & & 龚山美 & 50 \\
\hline & 钱敏之 & 441 & 1987 & 刘小祥 & 905 \\
\hline & 陈封怀 & 314 & & 陈龙清 & 482 \\
\hline & 刘克荣 & 153 & & 王少华 & 125 \\
\hline & 李洪均等 & 70 & & 饶均四 & 79 \\
\hline \multirow[t]{2}{*}{1959} & 吕志松 & 257 & & 舒金树等 & 73 \\
\hline & 赵子恩 & 156 & 1988 & 黄汉东 & 131 \\
\hline 1973 & 刘克荣 & 69 & 1991 & 赵子恩 & 221 \\
\hline \multirow[t]{5}{*}{1976} & 鄂神农架植考队 & 22511 & 1992 & 太阳坪考察队 & 1028 \\
\hline & 236-6 部队 & 973 & 1996 & 石世贵等 & 189 \\
\hline & 吴鹏程 & 391 & & 谭仲明\&韦若勋 & 60 \\
\hline & 周\&董 & 89 & 1997 & 谭策铭等 & 92 \\
\hline & 吴鹏程 & 76 & 2004 & 张宪春 & 125 \\
\hline 1977 & 鄂神农架植考队 & 2060 & 2008 & 喻勋林等 & 239 \\
\hline 1978 & 鄂神农架植考队 & 635 & 2011 & 张代贵 & 229 \\
\hline 1980 & 中美联合鄂西植考队 & 4861 & 2012 & 张代贵 & 497 \\
\hline & & & & 吴增源等 & 92 \\
\hline
\end{tabular}


谢丹, 王玉琴，张小霜，吴玉，张代贵。神农架自然遗产地植物标本采集史. 生物多样性，2019，27(2)：211-218. http://www. biodiversity-science. net/CN/10. 17520/biods. 2018255

\section{参考文献}

Chen YS (2012) A new species and a new combination in Parasenecio (Asteraceae). Annales Botanici Fennici, 48, 166-168.

Deng T, Kim C, Zhang DG, Zhang JW, Li ZM, Nie ZL, Sun H (2013) Zhengyia shennongensis: A new bulbiliferous genus and species of the nettle family (Urticaceae) from Central China exhibiting parallel evolution of the bulbil trait. Taxon, 62, 89-99.

Deng T, Zhang XS, Kim C, Zhang JW, Zhang DG, Volis S (2016) Mazus sunhangii (Mazaceae), a new species discovered in central China appears to be highly endangered. PLoS ONE, 11, e0163581.

Gao Q, Yang QE (2009) Aconitum shennongjiaense (Ranunculaceae), a new species from Hubei, China. Botanical Studies, 50, 251-259.

Ge XJ, Kirschner J, Štěpánek J (2011) Taraxacum F. H. Wiggers. In: Flora of China (eds Wu ZY, Raven PH, Hong DY), pp. 306. Science Press, Beijing \& Missouri Botanical Garden Press, St. Louis.

Hong DY, Kang YS, Shen LD (1983) Campanulaceae. In: Flora Reipublicae Popularis Sinicae, Tomus 73(2), (eds Yu DJ, Wu ZY, Cui HB), pp. 123, 186-187. Science Press, Beijing. (in Chinese) [洪德元, 康永善, 沈联德 (1983) 桔梗科. 见: 中国植物志第七十三卷第二分 册(俞德浚, 吴征镒, 崔鸿宾编), 123+186-187. 科学 出版社, 北京]

Jiang DS, Zhou PH, Chen DM (2000) Two new species of Pteridophytes from Shennongjia. Journal of Hunan Agricultural University, 26(2), 88-89. (in Chinese with English abstract) [蒋道松, 周朴华, 陈德惁 (2000) 神 农架蒴类植物二新种. 湖南农业大学学报, 26(2), 88-89.]

Kong XX, Wang PS (1997) New materials for the Cyrtomium Presl of China. Chinese Journal of Applied and Environmental Biology, 3, 23-25. (in Chinese with English abstract) [孔宪需, 王培善 (1997) 中国贯众 属的新资料. 应用与环境生物学报, 3, 23-25.]

Li B, Chen SF, Li Y, Wang DX, Du W (2016) Koenigia hedbergii (Polygonaceae: Persicarieae), a distinct new species from Shennongjia National Nature Reserve, Central China. Phytotaxa, 272, 115-124.

Li WP, Zhang ZG (2004) Aster shennongjiaensis (Asteraceae), a new species from Central China. Botanical Bulletin of Academia Sinica, 45, 95-99.

Li XD, Li JQ, Zan Y (2005) A new species of Triaenophora (Scrophulariaceae) from China. Novon, 15, 559-561.

Li XD, Zan YY, Liu HT, Li JQ (2011) A new form of Hepatica henryi (Oliv.) Steward. Acta Botanica Boreali-Occidentalia Sinica, 31, 2333-2334. (in Chinese with English abstract) [李晓东, 各艳燕, 刘宏涛, 李建 强 (2011) 川鄂獐耳细辛一新变型. 西北植物学报, 31, 2333-2334.]
Lin YR (1984) Materiae novae generis Artemisiae Chinensis (I). Bulletin of Botanical Research, 4(2), 14-34. (in Chinese and Latin) [林有润 (1984) 中国蒿 属植物新资料(I). 植物研究, 4(2), 14-34.]

$\mathrm{Mu}$ X, Xia X, Zhao L, Zhang Z (2012) Celastrus obovatifolius sp. nov. (Celastraceae) from China. Nordic Journal of Botany, 30, 53-57.

Pei YL, Hong DY (1995) Paeonia qiui-A new woody species of Paeonia from Hubei, China. Acta Phytotaxonomica Sinica, 33, 91-93. (in Chinese with English abstract) [裴颜龙, 洪德元 (1995) 卵叶牡丹一一苟药 属一新种. 植物分类学报, 33, 91-93.]

Shen ZH, Zhao ZE (2005) Arenaria shennongjiaensis, a new species of the Caryophyllaceae from Hubei, China. Acta Phytotaxonomica Sinica, 43, 73-75. (in Chinese with English abstract) [沈泽昊, 赵子恩 (2005) 湖北 无心菜属(石竹科)一新种——神农架无心菜. 植物分 类学报, 43, 73-75.]

Spongberg SA (1986) Notes on simple-leaved Chinese species of Sorbus (Rosaceae) from Hubei Province. Journal of the Arnold Arboretum, 67, 252-261.

Wang Q, Gadagkar SR, Deng HP, Yang ZM, Yu FQ (2016) Impatiens shennongensis (Balsaminaceae): A new species from Hubei, China. Phytotaxa, 244: 96-100.

Wang WC, Liu L, Wang SX, Zhang MZ, Ding ZZ, Ling PP, Fang MY, Tomus 28, (1980) Ranunculaceae. In: Flora Reipublicae Popularis Sinicae (eds Yu DJ, Wu $\mathrm{ZY}$, Cui HB), p. 355. Science Press, Beijing. (in Chinese) [王文采, 刘亮, 王蜀秀, 张美珍, 丁志遵, 凌苹苹, 方明渊 (1980) 桔梗科. 见: 中国植物志第 二十八卷 (俞德浚, 吴征镒, 崔鸿宾编), 355页. 北京, 科学出版社. ]

Wang WC (1981) Notulae de Gesneriaceis Sinensibus (II). Bulletin of Botanical Research, 1(4), 35-75. (in Chinese with English abstract) [王文采 (1981) 中国苦菅苔科 的研究(II). 植物研究, 1(4), 35-75.]

William TS (1996) Epimedium acuminatum and allied Chinese species (Berberidaceae). Kew Bulletin, 51, 396-398.

Wu ZY, Zhuang X (1990) A new section of Corydalis Sect. Davidianae. Acta Botanica Yunnanica, 12, 285-286. (in Chinese with English abstract) [吴征镒, 庄璇 (1990) 紫堇属一新组一一南黄堇组. 云南植物 研究, 12, 285-286.]

Xie D, Qian D, Zhang MH, Wang YQ, Wu Y, Huang LQ, Zhang DG (2017) Phytolacca exiensis, a new species of Phytolaccaceae from west of Hubei Province, China. Phytotaxa, 331, 224-232.

Yu DJ, Lu LT, Gu CZ, Li CL (1985) Taxa Nova Rosacearum Sinicarum (V). Acta Phytotaxonomica Sinica, 23, 209-215. (in Chinese with English abstract) [俞德浚, 陆玲娣, 谷粹芝, 李朝銮 (1985) 中国蓄薇 科植物分类之研究(五). 植物分类学报, 23, 209-215.]

Zhang L, Zhu ZM, Gao XF, Zhang LB (2013) Polystichum hubeiense (Dryopteridaceae), a new fern species from Hubei, China. Annales Botanici Fennici, 50, 107-110. 
谢丹, 王玉琴, 张小霜, 吴玉, 张代贵. 神农架自然遗产地植物标本采集史. 生物多样性, 2019, 27 (2)：211-218.

http://www. biodiversity-science. net/CN/10. 17520/biods. 2018255

Zhang YJ, Li JQ (2009) A new species of Epimedium (Berberidaceae) from Hubei, China. Novon, 19, 567-569.

Zhang YH (1986) A revision of genus Yinshania (Cruciferae). Acta Phytotaxonomica Sinica, 25, 204-219. (in Chinese with English abstract) [张渝华 (1986) 阴山荠属的校订. 植物分类学报，25, 204-219.]

Zhang YH (1996) A study on the genus Yinshania.
Bulletin of Botanical Research, 15, 364-365. (in Chinese with English abstract) [张渝华 (1996) 阴山 荠属的研究. 植物研究, 15, 364-365.]

Zhao B, Gong MX, Zhang QX (2007) A new variety and a new forma of Chimonanthus Lindl. from Shennongjia, China. Bulletin of Botanical Research, 27, 131-132. (in Chinese with English abstract) [赵冰, 龚梅香, 张启翔 (2007) 中国神农架蜡梅属一新变 种和新变型. 植物研究, 27, 131-132.] 